\title{
Téoros
}

Revue de recherche en tourisme

\section{Le complexe touristique de Marina d'Or à Oropesa del Mar} Un nouvel attracteur sur la Costa Azahar

\section{Carine Fournier}

Volume 27, numéro 2, été 2008

Les grands équipements touristiques

URI : https://id.erudit.org/iderudit/1070793ar

DOI : https://doi.org/10.7202/1070793ar

Aller au sommaire du numéro

Éditeur(s)

Université du Québec à Montréal

ISSN

0712-8657 (imprimé)

1923-2705 (numérique)

Découvrir la revue

Citer cet article

Fournier, C. (2008). Le complexe touristique de Marina d'Or à Oropesa del

Mar : un nouvel attracteur sur la Costa Azahar. Téoros, 27(2), 18-22.

https://doi.org/10.7202/1070793ar d'utilisation que vous pouvez consulter en ligne.

https://apropos.erudit.org/fr/usagers/politique-dutilisation/ 


\section{Le complexe touristique de Marina d'Or à Oropesa del Mar Un nouvel attracteur sur la Costa Azahar}

\section{Carine Fournier}

Initialement conçues autour d'une demande touristique axée sur le soleil et la plage, les stations balnéaires espagnoles n'offraient pas d'équipements complémentaires tels que des ports de plaisance ou des casinos comme on les trouvait dans des stations situées sur d'autres littoraux touristiques (Côte d'Azur, Languedoc-Roussillon en France). La question de l'alternative au sol y playa s'est donc posée avec acuité lorsque l'Espagne fut concurrencée par d'autres destinations plus attractives et moins coûteuses.

Oropesa del Mar, station balnéaire de la Costa Azahar, aménage actuellement, sous la houlette d'un industriel local, un projet touristique de grande envergure : Marina d'Or, ciudad de vacaciones [ville de vacances]. Ce projet, d'un genre nouveau pour l'Espagne, survient au milieu des années 1990. II ressemblait d'abord à une urbanización touristique traditionnelle, mais, en moins de 15 ans, il s'est transformé en mégaprojet touristique.

Avant de montrer comment, malgré une situation sur la partie la moins attractive (sable de galets et non pas sable fin) de la commune d'Oropesa del Mar, Marina d'Or s'est imposée comme une marque touristique en Espagne et en Europe, il convient d'évoquer le développement des stations touristiques espagnoles, pour bien remettre dans son contexte cette opération d'aménagement.

La question des impacts spatiaux et sociaux dus à l'implantation d'un complexe touristique de cette envergure sur une station balnéaire constituée autour du modèle sol y playa sera abordée, de même que les interrogations portant sur l'avenir des quartiers touristiques aménagés au cours des années. Ceux-ci sont-ils touchés par une baisse de fréquentation au profit de cet espace nouvellement urbanisé ?

\section{Un complexe touristique dans une station balnéaire de sol y playa}

L'Espagne, avec 59,2 millions de visiteurs en $2007^{1}$, se hisse à la deuxième place mondiale des pays touristiques, juste derrière la France (78 millions). En matière d'offre touristique, les principaux équipements de ce pays se concentrent sur les littoraux, en particulier sur la côte méditerranéenne. Les logements touristiques (appartements, villas, loués ou achetés) constituent le principal mode d'hébergement d'une grande partie des touristes (environ 70 \%). Le développement du tourisme littoral en Espagne date du milieu des années 1950. II a consisté en un aménagement dit spontané, c'est-àdire que les stations n'ont pas été planifiées par l'État espagnol : leur édification a été largement laissée à l'initiative privée.

La mise en tourisme est très rapide. Les premiers aménagements sont réalisés de façon anarchique, sans aucune marque légale, sans aucun plan. Dans certaines stations, des plans d'occupation des sols (PGOU plan general de ordenación urbana [plan général d'ordonnancement urbain]) sont approuvés environ sept à huit ans après la mise en place des principaux équipements. Dans les stations dotées d'un PGOU, les règles d'urbanisme édictées par ce dernier ne sont pas toujours respectées ; les communes ne font cependant pas détruire ces constructions illicites.
La forme urbaine qui résulte le plus souvent de ce mode d'aménagement est présentée comme étant l'alignement d'immeubles parallèles au front de mer, car la mise en tourisme de l'Espagne est passée par la construction d'appartements et non par un hébergement sous la forme d'hôtels ou de campings.

Le développement des playas ${ }^{2}$ sur les côtes espagnoles rappelle celui survenu sur les lidos adriatiques au début du XIX siècle avec l'apparition de la voie ferrée qui dessert toutes les stations balnéaires qu'elle coupe en deux : le lido et sa plage tournés vers la mer et l'arrièreplage où sont localisées les villes et servant surtout à l'espace résidentiel. " Le chef-lieu est localisé à la limite intérieure du cordon littoral, tandis que son satellite balnéaire ou lido proprement dit est directement installé sur le trait de côte sableux ", faisant apparaître un " espace ludique balnéo-urbain " (Lozato-Giotart 1993 : 124-125).

La publication, dans un ouvrage du sociologue Mario Gaviria (1975), des résultats d'une enquête menée auprès de touristes fréquentant les stations balnéaires espagnoles est à l'origine de la dénomination sol y playa pour caractériser le tourisme de bord de mer en Espagne. Elle démontre en effet que leur principale motivation est la recherche du soleil et de la plage (les touristes désignent alors la mer en troisième position). La nécessité de construire des équipements touristiques complémentaires n'apparait donc pas comme une évidence. En revanche, lorsque la fréquentation touristique des stations espagnoles chute vers la fin des années 1980 et le début des années 1990, l'enrichissement de l'offre touristique de ces dernières devient crucial. 


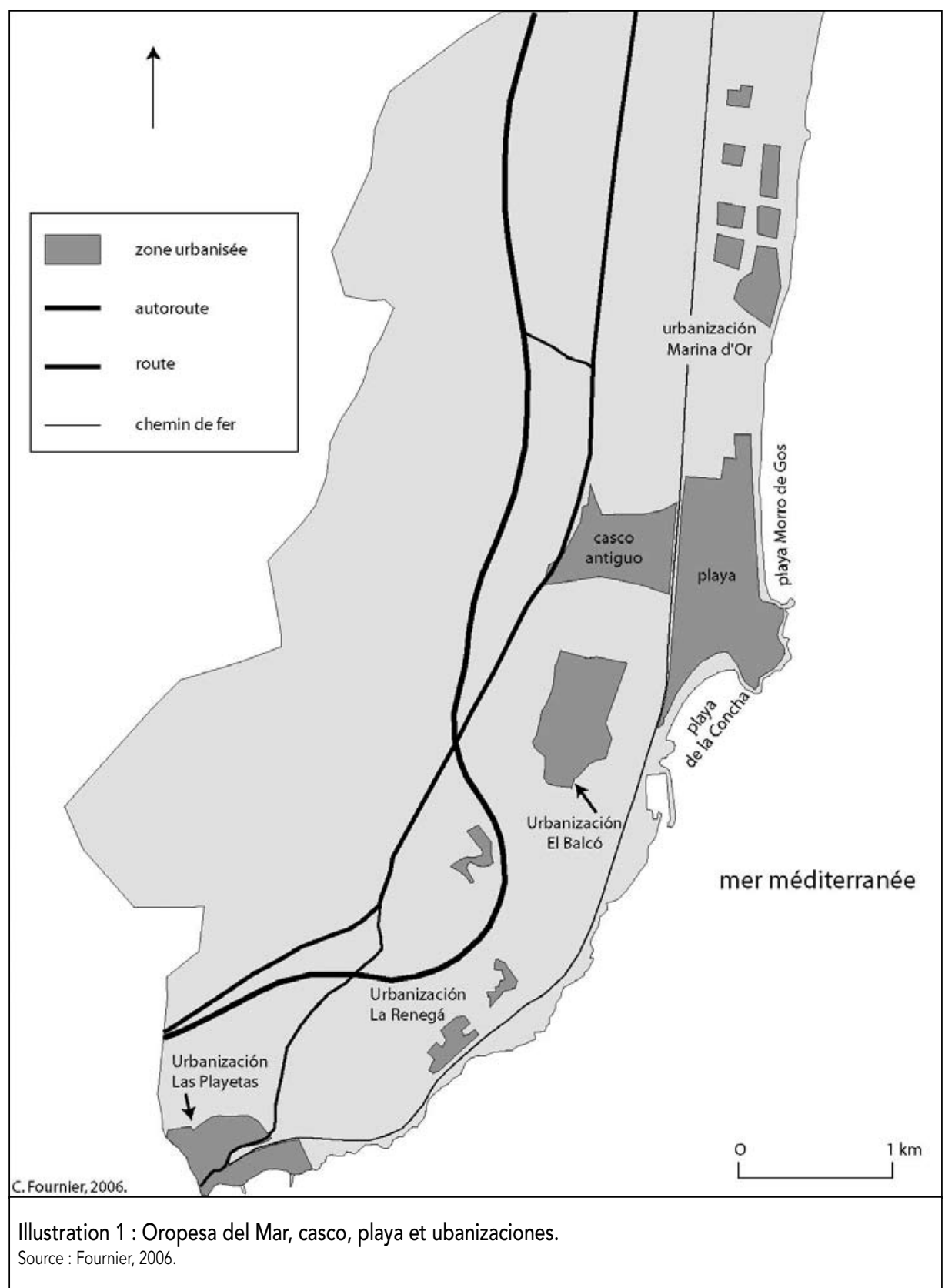

Les communautés autonomes, qui bénéficient alors, grâce à la Constitution de 1978, du transfert de compétences de l'État en matière de tourisme, se dotent d'une agence du tourisme. Ces agences sont créées dans le but d'améliorer la compétitivité et la qualité des produits touristiques proposés par chaque région ; des lois du tourisme sont aussi rédigées par ces administrations ${ }^{3}$.

Après avoir édité des livres blancs du tourisme espagnol mais aussi aux échelons régionaux, le ministère du Tourisme et les Communautés autonomes décident de structuraux qui ont pu être générés par l'absence de plan d'occupation du sol durant de trop nombreuses années. Les objectifs des plans d'excellence touristique sont de diversifier et d'enrichir l'offre, d'augmenter la qualité environnementale de la station en construisant par exemple des espaces verts, de proposer des produits qui correspondent aux demandes actuelles des touristes et de désaisonnaliser l'offre. Le premier plan d'excellence touristique est adopté à Calvià, aux Baléares.

La construction de ports de plaisance constitue le premier temps de la diversification de l'offre touristique en Espagne. D'autres voies sont suivies, notamment celle des parcs de loisirs thématiques. Cette option de restructuration des destinations touristiques balnéaires est plus particulièrement étudiée dans le cas de la Catalogne par Salvador Antón Clavé (1996 ; 1997 ; 1998), dans sa thèse de doctorat ainsi que dans nombre de ses écrits. II développe notamment l'exemple du parc thématique de Port Aventura à Salou, sur la Costa Dorada, dont l'ouverture en 1995 relance la fréquentation de la région.

L'implantation d'un complexe touristique à Oropesa del Mar constitue un cas quasi inédit sur les littoraux espagnols. Oropesa del Mar est une petite station de la Costa Azahar, située à 22 kilomètres de la capitale provinciale Castellón de la Plana. Comptant seulement 2500 résidents permanents au début des années 1990, cette station était assez peu connue (la station la plus réputée de la province est Benicasim, qui est directement jointive avec la capitale provinciale) jusqu'à ce qu'elle se trouve inscrite dans une aire de villégiature estivale fréquentée par les principaux décideurs politiques et industriels madrilènes (Rieucau, 2002). La venue du futur premier ministre José María Aznar dans l'urbanización Las Playetas, située sur la côte rocheuse de la commune, donne une renommée nationale à Oropesa del Mar. Selon Jean Rieucau (2002 : 38), il y a alors une réinvention du lieu touristique. José Soriano, président-directeur de la firme Porcelanosa, met ainsi sa villa de bord de mer à la disposition de José María Aznar. Les milieux financiers et industriels sont présents à Las Playetas, notamment 
Juan Villalonga, ancien président de Telefónica, mais aussi Carlos Fabra, président du conseil provincial de Castellón. Tout cela confère une notoriété soudaine à cette commune restée jusque-là dans l'ombre de Benicasim.

C'est dans ce contexte que Jesus Ger, un entrepreneur de Castellón de la Plana spécialisé dans la fabrication de matelas, décide de faire sortir de terre un complexe touristique à Oropesa del Mar, avec l'appui de la municipalité et de son ami Carlos Fabra.

\section{Un comptoir touristique de grande envergure}

Le complexe touristique de Marina d'Or est situé au nord de la commune d'Oropesa, en bord de mer, en discontinuité totale avec l'urbanisation de la station (ill. 1). Cet éloignement du quartier touristique traditionnel, aménagé à partir des années 1960, marque bien la rupture souhaitée par Jesus Ger, tant spatialement qu'architecturalement. La recherche de la plage de sable fin n'étant plus tout à fait une priorité pour les touristes, la localisation de Marina d'Or à proximité d'une plage très étroite de galets n'apparaît pas comme un handicap pour la réussite du projet.

Les premiers plans et les premiers documents touristiques fournis et distribués aux acheteurs potentiels font état d'appartements, d'une zone commerciale et de la présence d'un hôtel trois étoiles accompagné d'un centre de thalassothérapie. Rien de bien différent de ce que l'on peut trouver dans certaines urbanizaciones du pays, voire de la commune. Pourtant, les ambitions de Marina d'Or sont tout autre. En effet, Jesus Ger tente d'imposer son complexe telle une véritable « bulle » à l'intérieur de la commune.

L'idée de Marina d'Or est de proposer un lieu de repos à des familles, et ce durant toute l'année. Mais le succès n'est pas assuré, le projet voulant aussi répondre aux besoins de tous ses clients qui ne souhaitent pas se rendre dans le casco antiguo, ni sur la playa. Marina d'Or apporte tout le nécessaire et surtout le superflu. Effectivement, rien ne manque à Marina d'Or. On y trouve des commerces (restaurants, cafeterias, coiffeurs...), un parc de loisirs où les enfants peuvent s'amuser tandis que les parents se reposent au jacuzzi. L'hôtel étant toujours ouvert, les appartements peuvent être loués toute l'année, mais il offre aussi des séjours à la carte.

En juin 2001, de nouvelles brochures sont distribuées. Outre le centre de thalassothérapie dont les mérites sont vantés, on trouve à présent un vaste programme immobilier de plusieurs milliers d'appartements dont les immeubles répondent aux noms d'Acapulco, de Bahía Park et d'Isla Park.

La zonation spatiale du complexe touristique apparaît comme relativement simple, mais particulièrement efficace. L'hébergement hôtelier est concentré au sud de l'urbanisation, autour du centre de balnéothérapie. S'y déploient cinq hôtels classés de trois à cinq étoiles. Notons que l'hôtel cinq étoiles avec ses 184 chambres fait partie des rares hébergements de ce standing dans la communauté de Valence. Les appartements sont, quant à eux, situés plus au nord et s'étendent jusqu'aux limites septentrionales de la commune. Ici, point d'immeubles de plus de 15 étages, comme cela peut être le cas dans le quartier balnéaire le plus ancien de la commune. La zone commerciale la plus fournie se situe autour du complexe hôtelier.

La végétalisation de l'ensemble tranche avec le reste de la station balnéaire. Des jardins y sont en effet aménagés. De grands espaces de promenades sont aussi prévus. Le complexe touristique propose une paseo marítimo, haut lieu de l'urbanité espagnole. Cette promenade est d'ailleurs fréquentée par l'ensemble de la population permanente de la station.

À compter de 2001, Marina d'Or guide l'urbanisation de la commune d'Oropesa plus au nord. Marina d'Or regarde en direction de la commune voisine, celle de Cabanes, et surtout vers les campings de la commune. Depuis, ces hébergements touristiques ont été entièrement rasés. Le nouveau projet en cours, Marina d'Or golf, se situe à la fois sur la commune d'Oropesa et sur celle de Cabanes. II est conçu pour accueillir 100000 touristes et devrait s'étendre sur près de 19 millions de mètres carrés. Jesus Ger prévoit la construction de 50000

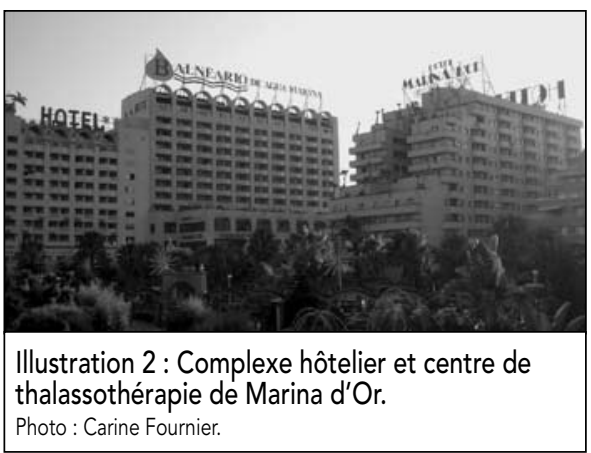

logements, un ski park (pour skier face à la Méditerranée) ainsi que trois parcours de golf, dont un créé par Sergio García et Greg Norman (une sommité dans le domaine).

À ces projets, il convient d'ajouter un parc à thème, sur la magie : Mundo llusión. La construction de ce dernier sera entièrement prise en charge par le conseil provincial de Castellón et par la communauté de Valence, fait rarissime dans une région où la plupart des investissements touristiques relèvent, depuis le milieu des années 1950, du secteur privé. Le complexe de Marina d'Or est particulièrement bien relié au reste de l'Espagne grâce à l'autoroute A7 et à une nouvelle sortie de cette dernière, réalisée justement pour en faciliter l'accès. II devrait bientôt être relié à l'Europe entière grâce à l'aéroport de Castellón. Celui-ci, qui se dénomme Castellón-Costa Azahar, est très souvent rebaptisé Castellón-Marina d'Or par les opposants au projet, car l'emplacement choisi se trouve à 15 kilomètres de l'urbanización touristique.

La dénomination Marina d'Or est toujours suivie de l'expression ciudad de vacaciones, qui signifie "ville de vacances". Le séjour du touriste à Marina d'Or doit ainsi être vécu comme une véritable rupture avec le quotidien. Dans l'idée de son concepteur, le visiteur qui se rend à Oropesa pour être hébergé à Marina d'Or ne doit plus avoir à se servir de sa voiture ; tous les services doivent être à portée de main et faciliter sa détente. Marina d'Or correspond bien à la définition d'un complexe touristique classique tel que Daniel Clary (1993) l'évoque : " une cellule de vie complète, autonome avec ses équipements, ayant ciblée [sic] un ou quelques objectifs d'activités, un certain style de vacances, et par conséquent un certain segment de clientèle ». 


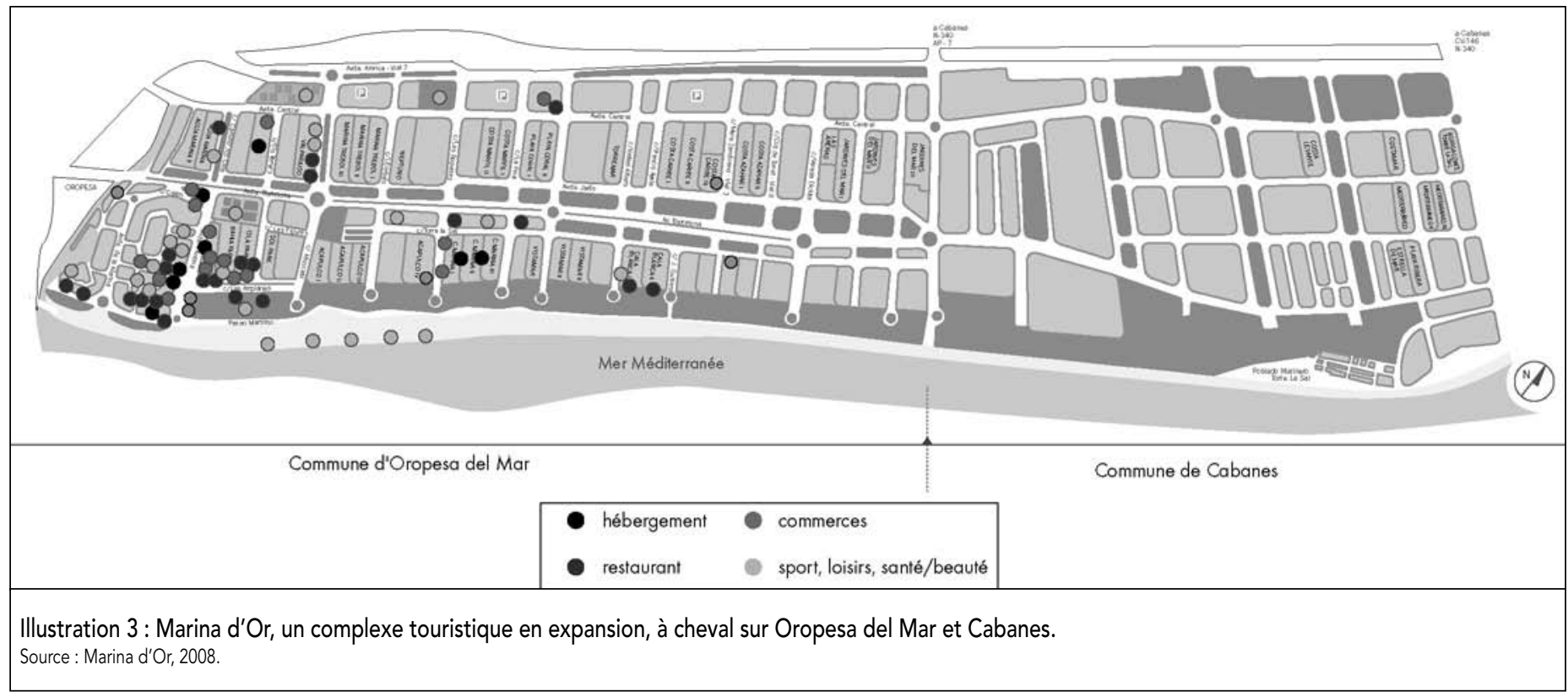

À sa conception, au regard des classifications des lieux touristiques formulées par les géographes français, Marina d'Or pouvait donc être assimilée à un comptoir touristique. En effet, le comptoir, tel qu'il a été décrit par Rémy Knafou (1997), puis par l'Équipe MIT $(2002,2005)$ et sous la forme d'un manuel (Stock, 2003), se définit par la " primauté de l'activité touristique dans le lieu, mais c'est un lieu créé ex nihilo pour accueillir des touristes deux ou trois jours, une ou deux semaines ". Rémy Knafou ajoute que les comptoirs touristiques sont déterritorialisés et qu'ils n'entretiennent que des relations occasionnelles avec leur environnement; en aucun cas ils ne sont des lieux de vie; ils se limitent à une fonction d'hébergement et ne possèdent pas de fonction de résidence. Ils sont les produits de ce qu'on appelle parfois encore "l'industrie touristique " et ils ne prétendent aucunement devenir des villes dotées d'une population permanente et de fonctions économiques autres que touristique.

Si un comptoir touristique n'a pas vocation à devenir une ville ou un quartier de station, quels sont les impacts sociaux et spatiaux qu'il peut avoir, à terme, sur l'espace dans lequel il s'insère ? Permet-il de soutenir l'activité touristique déjà existante dans le lieu ou bien conduit-il à la faire péricliter?

\section{Marina d'Or, un complexe touristique créateur d'urbanité}

Le premier hôtel de Marina d'Or ainsi que le centre de balnéothérapie ont ouvert leurs portes au public en 1999. Après un peu plus de huit années de fonctionnement, la réussite du projet semble se confirmer.

La stratégie publicitaire mise au point par Jesus Ger a probablement joué un rôle important dans ce succès. En effet, celle-ci repose tout d'abord sur une sorte de matraquage médiatique, notamment par la diffusion en boucle, en Espagne mais aussi en France et dans d'autres pays européens, aux heures de grande écoute, d'un spot publicitaire vantant les mérites du « plus grand centre de balnéothérapie d'Europe ». Ensuite, un formidable réseau de bureaux et d'agences maille les territoires espagnols et européens, permettant de toucher tous les segments possibles de population. On dénombre pas moins de 89 agences en Espagne réparties sur 35 provinces. Elles couvrent ainsi quasiment la totalité du pays. À l'étranger, ce sont la France (une agence à Paris), l'Angleterre (une agence à Londres) et la Russie (une agence à Moscou) qui sont au cœur des initiatives destinées à positionner la ville de vacances et à redorer le blason d'une région qui a connu la désaffection des touristes de l'Europe de l'Ouest depuis la fin des années 1990.
Mesurée en termes de fréquentation touristique, la réussite de Marina d'Or est en effet incontestable. Ce succès se calcule tout d'abord en nombre de nuitées puisque, par exemple, on en a enregistré pas moins de 500000 pour l'année 2005. Les bénéfices avant impôts, pour cette même année, ont atteint 108 millions d'euros. Le taux des réservations pour la semaine sainte de l'année 2008 a été de $95 \%$ des 2000 places d'hôtel disponibles ${ }^{4}$. Durant la période estivale, le taux d'occupation des hôtels d'Oropesa avoisine les $100 \%$ et la fréquentation touristique ne cesse d'augmenter depuis 2001. Le développement de Marina d'Or a donc permis une reprise de l'activité touristique dans la station.

Le rythme de vente des appartements est, quant à lui, très soutenu. Sur la période allant de 2004 à 2006, 1500 appartements ont été vendus en moyenne par an dans le quartier de Marina d'Or. L'année 2007 a par ailleurs été moins propice aux transactions, puisque seulement 400 appartements ont été vendus.

On pourrait logiquement penser que cette vente d'appartements est uniquement destinée à la résidence secondaire et que les appartements seront occupés seulement quelques mois dans l'année, préférablement durant la haute saison. Or, et même si la tendance est effectivement 
d'acheter des résidences destinées à la villégiature estivale, dès 2001, alors que le quartier de Marina d'Or n'offrait pas autant de logements qu'aujourd'hui (il était alors en pleine construction) et que les commerces n'étaient pas aussi nombreux et ouvraient seulement une partie de l'année, une population permanente était déjà présente. Cela représentait une centaine de personnes et 50 ménages qui avaient décidé de s'y établir et d'y résider à l'année. Un ouvrage de 1990 de Francisco Jurdao Arrones intitulé España, asilo de Europa montre comment des retraités venaient s'établir dans les stations balnéaires du pays. Aujourd'hui, les personnes qui s'installent dans des stations balnéaires ne sont pas uniquement des retraités comme a pu le démontrer Philippe Duhamel (1997) dans sa thèse de doctorat. Ce constat est avéré pour Oropesa del Mar ainsi que pour Marina d'Or ; en effet, parmi les résidents permanents, seulement $3 \%$ sont âgés de plus de 65 ans dans ce quartier. La tranche d'âge la plus représentée est celle des 25-45 ans.

$\mathrm{Au}$ recensement de 2001 (Instituto Nacional de Estadistica, 2001), Oropesa del Mar comptait 4287 habitants. Au $1^{\text {er }}$ janvier 2007, la population permanente de la station balnéaire était passée à 9613 habitants. On ne connaît pas la répartition exacte par quartiers, mais la population permanente à Marina d'Or a augmenté si I'on en juge par les allées et venues au sein de I'urbanización. Depuis 2002, le complexe touristique est d'ailleurs dénommé el pueblo nuevo [le nouveau centre] par la population locale. Le terme pueblo étant généralement réservé au centre ancien, cette désignation montre à la fois que le quartier est actif toute l'année et qu'il est un lieu où la population se retrouve.

Tandis que Jesus Ger s'évertue à faire de Marina d'Or une bulle touristique au sein d'une station balnéaire, celle-ci se transforme en centre névralgique de la station. C'est d'ailleurs ce complexe touristique qui guide dorénavant l'urbanisation de la commune puisque la plupart des édifices construits le sont dans cette partie. Les installations nouvelles s'y font en priorité, proposant des commerces ouverts à l'année et de très nombreux services à la personne.

\section{Conclusion}

Marina d'Or - Ciudad de vacaciones, présentée, à ses débuts, comme un équipement touristique complémentaire d'une offre totalement centrée sur le sol y playa, s'est peu à peu transformée en véritable bulle touristique, offrant des services qu'aucune station de la Costa Azahar n'a jusqu'alors été en mesure de proposer aux touristes.

Ce projet touristico-immobilier connaît un succès incontestable. II attire non seulement des touristes venus pour voir le lieu touristique à la mode, celui que fréquentait et continue de fréquenter l'ancien premier ministre espagnol José Maria Aznar, mais aussi des familles venues pour voir de leurs yeux le mégaprojet touristique qui leur est proposé chaque soir aux heures de grande écoute sur les chaînes télévisées nationales.

Or, ce qui apparaît comme le plus intéressant, c'est que là où les concepteurs pensaient créer un monde à part, une bulle de vacances, ils sont parvenus à créer un nouveau quartier dans la commune, un lieu de vie, animé à l'année et où la population permanente ne cesse de croître. Le complexe touristique de Marina d'Or apparaît donc comme créateur d'urbanité dans la station touristique d'Oropesa del Mar, marquée jusque-là par un fonctionnement saisonnier.

Carine Fournier, docteure en géographie, est chargée de cours à l'Université Lumière - Lyon 2.

\section{Notes}

1 [http://www.world-tourism.org].

2 Playa est le terme utilisé en Espagne pour désigner le quartier de bord de mer d'une commune littorale.

3 Les attributions de l'Agence du Tourisme de la Communauté de Valence ainsi que la Loi sur le tourisme au sein de cette même entité administrative peuvent être consultées sur le site : [http://www.comunidad-valenciana. org/].

4 Quotidien régional Las Provincias, édition du 15 mars 2008.

\section{Bibliographie}

Antón Clavé, Salvador (1996), «El parque temático Port Aventura. Estrategia de producto para la reestructuración de núcleos turísticos consolidados en Cataluña (el papel de la administración como motor del cambio en Salou y Vila-Seca) " [Le parc thématique Port Aventura. Stratégie pour la restructuration des destinations touristiques consolidées en Catalogne (le rôle de l'administration comme moteur du changement à Salou et Vila-Seca)], Estudios turísticos [Études touristiques], n 130, p. 7-36.

Antón Clavé, Salvador (1997), Diferenciació i reestructuració de l'espai turístic. Processos i tendències al litoral de Tarragona [Différenciation et restructuration de l'espace touristique. Processus et tendances sur le litoral de Tarragone], Tarragona, Edicions el Mèdol.

Antón Clavé, Salvador (1998), " La urbanización turística. De la conquista del viaje a la reestructuración de la ciudad turística " [L'urbanisation touristique. De la conquête du voyage à la restructuration de la ville touristique], Documentos de analisis geografica [Documents d'analyse géographique], $\mathrm{n}^{\circ} 32$, p. 17-43.

Clary, Daniel (1993), Le tourisme dans l'espace français, Paris, Masson.

Duhamel, Philippe (1997), Les résidents étrangers européens à Majorque (Baléares) : pour une analyse de la transformation des lieux touristiques, Thèse de doctorat, Université Paris VII.

Équipe MIT (2002), Tourismes 1, Lieux communs, Paris, Belin.

Équipe MIT (2005), Tourismes 2, Moments de lieux, Paris, Belin.

Fournier, Carine (2006), Entre station et ville : une ægialopolis ? L'Espagne et ses costas : Costa Azahar et Costa Valencia, thèse de doctorat, Université Montpellier 3.

Gaviria, Mario (1975), El turismo de playa en España - Chequeo a 16 ciudades nuevas de ocio [Le tourisme de plage en Espagne Étude de 16 villes nouvelles de loisir], Madrid, Turner.

Instituto Nacional de Estadistica (2001), Censos de población y viviendas 2001, resultados definitivos [Recensement de la population et des logements 2001, résultats définitifs], [http://www.ine.es], consulté en février 2004.

Jurdao Arrones, Francisco (1990), España, asilo de Europa [Espagne, asile de l'Europe], Barcelona, Planeta.

Knafou, Rémy (1997), Atlas de France - Tourisme et loisirs, Paris, La documentation française.

López Olivares, Diego (1990), Espacio turístico $y$ residencial en las tierras castellonenses [Espace touristico-résidentiel sur les terres de Castellón], Castellón de la Plana, Sociedad Castellonense de Cultura.

Rieucau, Jean (2002), "Invention et création de lieux touristiques dans la dynamique de la diffusion urbaine littorale, au nord de la Costa del Azahar ", Cahiers de géographie du Québec, n 127, avril, p. 25-48.

Stock, Mathis (dir.) (2003), Le tourisme : acteurs, lieux et enjeux, Paris, Belin. 